\title{
Solving the over segmentation problem in applications of Watershed Transform
}

\author{
Mariela Azul Gonzalez ${ }^{1}$, Gustavo Javier Meschino ${ }^{2}$, Virginia Laura Ballarin ${ }^{1}$ \\ 1. Grupo de Procesamiento de Imágenes.Departamento de Electrónica. Facultad de Ingeniería. Universidad Nacional de \\ Mar del Plata, Argentina. 2. Laboratorio de Bioingeniería. Departamento de Electrónica. Facultad de Ingeniería. \\ Universidad Nacional de Mar del Plata, Argentina.
}

Correspondence: Mariela Azul Gonzalez. Address: Catamarca 1440 6to J, Mar del Plata, Buenos Aires, Argentina. Telephone: 54-223-156-077-108. E-mail: mazulgonzalez@fi.mdp.edu.ar.

Received: November 1, 2012 Accepted: February 27, 2013 Online Published: April 9, 2013

DOI : $10.5430 / j b g c . v 3 n 3 p 29$

URL: http://dx.doi.org/10.5430/jbgc.v3n3p29

\section{Abstract}

Background: The Watershed Transform consists of an image partitioning into its constitutive regions. This transform is easily adapted to be used in different types of images and it allows distinguishing complex objects. However, the implementation of the Watershed Transform for very complex images actually produces over-segmentation. In this paper we propose two algorithms to solve this over-segmentation problem.

Methods: We define internal markers, by algorithms based on clustering and fuzzy logic in order to join the oversegmented regions with statistical features. To define the algorithm parameters and evaluate their performance, errors against images segmented manually were measured and ROC curves were determined.

Results: The results show that the proposed methods self-adapt to the different image objects characteristics. An improvement of the accuracy is obtained.

Conclusions: This analysis will contribute in images segmentation where complexity of the objects is high.

\section{Key words}

Watershed transform, Over segmentation, Fuzzy logic, Clustering, Textured images

\section{I ntroduction}

The Watershed Transform (WT), a morphological tool, consists of an image partition into regions. The use of the WT in complex images, typically textured images, often ends up in over-segmentation ${ }^{[1,2]}$. In order to obtain regions that belong to the objects of interest, marker definition algorithms are necessary. These markers allow the initiation of the watershed flooding. Several marker definition algorithms have been proposed in order to improve segmentation results. Conventional threshold algorithms, region growing methods and mathematical morphology operators were applied without obtaining satisfactory results in complex image segmentation ${ }^{[3]}$. Moreover, region merging methods were also applied during a posterior stage ${ }^{[4]}$ without satisfactory results.

To determine internal markers, different algorithms were developed. One of these determines some features of the over-segmented regions and is based on Fuzzy Logic. Fuzzy Logic formalizes inaccurate, ambiguous knowledge, 
expressed linguistically ${ }^{[5,6]}$. Unlike Boolean Logic, where the only truth values are zero (false) and one (true), in Fuzzy Logic the truth values are all the real numbers into the $[0,1]$ interval. Assignation of the truth values is done by membership functions. These functions assign to each value of the variables, a membership degree for a linguistic concept (a fuzzy term). A fuzzy predicate is determined in order to describe whether a region is part of an object. The resulting degree of truth of this predicate is used to get internal markers for application of the WT. Flooding begins from these markers or centers.

Medical images present an inherent ambiguity, where resolution, definition, and contrast depend upon the user and each particular application. This generates blurry boundaries, textured objects and non-homogeneous regions, which makes the fuzzy logic, the more adequate application for that type of images. Obtaining a whole object is not necessary to define watershed markers; a good approximation of the object is enough to achieve satisfactory results. This proposed algorithm has a very low computational cost. This is very interesting when processing high resolution images ${ }^{[7-9]}$, as are bone marrow biopsies images. This proposal results efficient to encompass the knowledge of the experts. It is a semi-automatic segmentation, because it requires that the expert mark the object of interest.

In the second algorithm, the features of over-segmentation regions are determined by regional minima and the internal markers are selected using a clustering algorithm to join regions. The first five moments of each region were tested, but only mean value and standard deviation resulted in correct segmentations. WT markers are selected as the cluster that represents the objects of interest. Finally WT is applied again using these new markers. This proposal allows an effective and robust segmentation of highly textured images. Computational cost is lower than the one obtained with the first algorithm and the segmentation method is automatic ${ }^{[9]}$. On the other hand, it results in a less accurate segmentation in most of cases.

The markers of the background, external markers, were obtained by eroding the internal markers complements in both algorithms. This process results in adequate segmentations when internal markers sizes are similar to the size of the objects ${ }^{[9]}$. Generally in medical images, the objects border does not have a visible contrast. That adds another difficulty when applying the flooding algorithms. Consequently, obtaining internal and external markers of a considerable size, results in a segmentation improvement. The use of the proposed algorithms, developed from the over-segmentation, allows the determination of better markers and, as consequence, better segmentation.

Since these algorithms require several parameter definitions, in this work we propose an error analysis to adjust these parameters and improve medical image segmentation. This analysis will contribute in medical image segmentation, where the motivation of this work development relies.

\section{Subjects and methods}

\subsection{Watershed Transform}

A gray level image can be interpreted as the topographic image. It can be assumed that the gray intensities of higher magnitude correspond to mountains, whereas those of lesser value are consistent with valleys or rivers. Using this point of view, the WT is defined ${ }^{[10,11]}$. These transform recognizes the outlines of similar topographic regions delimited by mountain ridges through the flooding of valleys.

The first step for Transform application is to obtain the image gradient. Here, the boundaries of the objects are represented by higher gray levels. The zones of lower levels result in basins where the water would flow and flood the topography of the image. As a consequence, the objects contours would remain and give way to the segmentation of the image through the resulting Watershed lines. 
In the classic WT the image gradient is flooded from the regional minima. When dealing with images that have a non-homogenous noise, more than one minimum for each object maybe present, leading to over-segmentation ${ }^{[9]}$. Therefore, it is required to eliminate irrelevant boundaries through region merging algorithms.

\subsection{I nternal marker definition by fuzzy logic}

Fuzzy Logic is a valuable tool for the formalization of ambiguous linguistically expressed knowledge. Fuzzy predicates were proposed to design classification systems. Each possible class is described in different predicates. The class presenting the bigger degree of truth is assigned to the input.

A fuzzy predicate $p$, is a linguistic expression (a proposition) with degree of truth $\mu_{p} \in[0,1]$. The degree of truth of the predicate, $\mu_{p}$, can be obtained by the membership function associated with a fuzzy term. E.g.: the predicate "Intensity is high", is associated with the variable "intensity" which is measured in meters, and the concept "high" which is defined by a membership function over the magnitude of the intensity.

A compound predicate, $c p$, is a fuzzy predicate obtained by combination of simple fuzzy predicates or other compound fuzzy predicates, joined by logical connectives and operators (and, or, not). To compute the degree of truth of a compound predicate, it is necessary to define logic systems based on a quadruplet of continuous operators (conjunction, disjunction, order and negation). The definition is done over a set of truth values such that when the truth values are restricted to $\{0,1\}$, these operations become classic Boolean predicates.

Some logic systems are quite simple, for example the use of minimum and maximum operations for conjunction and disjunction, respectively. On the other hand, Compensatory Fuzzy Logic (CFL) is based on the removal of some axioms in order to achieve a sensitive and idempotent multi-valued system. These systems are sensitive to the predicates' degrees of truth involved, and they have been widely used to represent knowledge as a predicates system.

In the CFL based systems, conjunction and implication compensate low truth values with high truth values that belong to dissimilar variables, which make them suitable for modeling subjective knowledge. Therefore, the use of the CFL is analyzed, together with classic fuzzy operators, to address the complex task of grouping the over-segmented regions of the $\mathrm{WT}^{[5,12]}$. In addition, the same analysis is done by using probabilistic operators:

Given $\mathrm{n}$ truth values $X_{i}$, the CFL conjunction is determined as follows:

$$
c\left(x_{1}, x_{2}, \ldots ., x_{n}\right)=\left(x_{1} \cdot x_{2} \ldots . . x_{n}\right)^{1 / n}
$$

while probabilistic conjunction is defined as

$$
c\left(x_{1}, x_{2}, \ldots, x_{n}\right)=x_{1} \cdot x_{2} \ldots x_{n}
$$

CFL disjunction is determined as:

$$
d\left(x_{1}, x_{2}, \ldots, x_{n}\right)=1-\left[\left(1-x_{1}\right)\left(1-x_{2}\right) \ldots\left(1-x_{n}\right)\right]^{1 / n}
$$

and probabilistic conjunction for two operators is defined as:

$$
d\left(x_{1}, x_{2}\right)=x_{1}+x_{2}-x_{1} \cdot x_{2}
$$


Negation of a truth value ' $\mathrm{X}$ ' for both logics is defined as:

$$
n(x)=1-x
$$

In both cases, the degree of truth of the implication $x \rightarrow y$ can be computed with next expression, based on conjunction and disjunction:

$$
i(x, y)=d[n(x), y]
$$

Several methods were found that involve Fuzzy Logic segmentation to the histogram entropy function ${ }^{[10,13-16]}$. However, in textured medical images it is not possible to discriminate objects automatically by histogram segmentation. Moreover, its high variability in terms of shape and size makes mathematical morphology techniques insufficient.

In previous works, conventional Fuzzy Logic was employed to develop a Mamdani Fuzzy Inference System ${ }^{[7,8]}$. This algorithm assigns a level of membership to each class for each pattern instead of offering a unique grouping of them.

In the present paper, we propose the determination of a fuzzy predicate degree of truth computed by compensatory logic. In this way, we decide, in a fuzzy basis, whether a region of the image corresponds to an object of interest.

\subsection{I nternal marker definition by clustering}

In this method, we propose to apply clustering after over-segmentation to obtain watershed internal markers. Features extracted from over-segmentation resulting regions, are used as input to a clustering algorithm. This result is used to obtain the markers for a new application of the WT.

There are different methods that group these regions to segment images; however these methods usually don't consider the regions to obtain the markers for the $\mathrm{WT}^{[3]}$. These new algorithms were developed in order to reduce the sensitivity to noise and to increase the segmentation robustness of complex images with different features. We used the $k$-means algorithm. Fuzzy C-means algorithm was not used because it presented a lower accuracy in previous experiments. $\mathrm{K}$-means requires the specification of the number of classes in which the data set is going to be partitioned. The algorithm gives $\mathrm{K}$ cluster centers so that the distance of each pattern to its center is minimal. Initial cluster centers were fixed for all images in order to have always the same class label for the object of interest. The partition is done by measuring, in an iterative process, the distance between each pattern to its cluster center, and re-computing the centers as the mean between all data belonging to the same cluster, until there is no change ${ }^{[17]}$.

\subsection{Proposed algorithms}

As we mentioned before, the application of WT often produces an over-segmented image. To solve this problem, two different ways for determining proper internal marker definitions are proposed in this section. The first algorithm needs a trained expert to select some regions that correspond to the objects of interest by mouse clicking. After, basic statistical features of each region of the over-segmented image are computed, and internal markers are found to compute the marker-based WT. The second proposed algorithm is fully automatic.

\subsubsection{Fuzzy logic proposed algorithm}

Given an image presenting objects of interest, they can be segmented using WT by following the next steps:

Step 1. First WT. 
Preliminary regions are obtained by the computation of WT, using regional minima as markers. An over-segmented image is get, which has a big number of regions $R_{k}, k=1,2 \ldots m$, being $m$ the number of regions obtained. A label matrix is also obtained, where each pixel has a label assign to indicate the regional minimum to which it belongs.

Step 2. Statistical features computation.

Average and standard deviations of the gray level values of the pixels that belong to each region are computed and stored.

Step 3. Selection of object regions.

A trained expert selects a determined number $(n)$ of regions corresponding to the objects of interest. The number of regions to construct the membership functions is chosen heuristically based on previous tests. This number is a setting parameter and may differ from one type of image to another. Let's call the regions selected $S_{i}, i=1,2 \ldots n$.

Step 4. Construction of membership functions.

Features from selected regions are used to define membership functions. Gaussian-shaped functions with mean value and standard deviation computed in the previous step are used. One membership function is obtained for each object region.

Step 5. Computation of the degrees of truth.

This is the central step of the algorithm. A fuzzy predicate is defined to detect the regions that correspond to the objects of interest. For each region $R_{k}, k=1,2 \ldots m$, the degree of truth for the next predicate is computed:

$$
p_{k}=\text { "The region } R_{k} \text { belongs to an object of interest."; } k=1,2 \ldots m
$$

which is equivalent to say:

$$
\begin{aligned}
p_{k}= & \text { "The region } R_{k} \text { presents gray levels similar to } S_{1} \text { " } \\
& \text { or "The region } R_{k} \text { presents gray levels similar to } S_{2} \text { " } \\
& \text { or } \ldots \\
& \text { or "The region } R_{k} \text { presents gray levels similar to } S_{n} \text { " }
\end{aligned}
$$

symbolically written as:

$$
p_{k}=s_{1} \vee s_{2} \vee \ldots \vee s_{n}
$$

where $s_{i}=$ "The region $R_{k}$ presents gray levels similar to $S_{i}$ ".

The truth value of each simple predicate $S_{i}$ is computed via the truth values of the membership functions defined in the previous step.

The truth value of the main predicate $p_{k}$ for each region $R_{k}$ is computed by operating applying fuzzy operators (CFL and probabilistic) over the truth values of the simple predicates. This value is assigned pixelwise according to the region that each pixel belongs to. This procedure allows the creation of an intensity image, which has intensities between 0 and 1 . 
Step 6. Thresholding.

In order to obtain an image where only the objects of interest appear, a conventional thresholding with OTSU threshold ${ }^{[18]}$ is done. This is the internal marker image.

Step 7. Creation of external markers.

Markers for background, external markers, are obtained eroding the resulting image as the complement of the internal markers image.

This procedure results in adequate segmentations when of the internal markers sizes are similar to the objects sizes. The gradient of complex images does not have a visible contrast, making difficult the application of flooding algorithms directly. Consequently, by obtaining internal and external markers of a proper size, results are considerably improved.

\subsubsection{Clustering based algorithm}

After obtaining different regions by the applicatrion of the Watershed Transform to the original image, we propose the application of a non-supervised simple clustering algorithm. This algorithm takes mean and standard deviation of regions as data features.

This method can be summarized by next steps:

\section{Step 1. First WT.}

Preliminary regions are obtained by the computation of WT, using regional minima as markers. An over-segmented image is get, which has a big number of regions $R_{k}, k=1,2 \ldots m$, being $m$ the number of regions obtained. A label matrix is also obtained, where each pixel has a label assign to indicate the regional minimum to which it belongs.

Step 2. Median filtering.

A median filter is applied. We tried Gaussian filters and histogram equalization based filters, without noting significant differences. The filter mask size is heuristically chosen. The size of over-segmented regions depends on this filter.

Step 3. Features computation.

Features are computed for each region: average values and standard deviations of gray level values from the pixels that belong to each region. A $n \times 2$ data matrix is obtained, being $n$ the number of regions and the 2 columns the mean and standard deviation of each region, respectively.

Step 4. Clustering.

Data matrix is used as input for $k$-means algorithm. A 2 classes partition of the data set will be obtained. One of the clusters corresponds to object regions and the other corresponds to background regions. This gives a segmented image, with regions labeled according to the cluster index assigned by $k$-means.

Step 5. Morphological operations.

Morphological opening and closing operators are applied to obtain the object markers, internal markers. These operators join adjacent regions, which correspond to the objects of interest, and eliminate regions that do not belong to them. Then 
slight morphological erosion is applied in order to discard regions that belong to the objects edges. The obtained image will be used as internal markers for the Watershed Transform.

Markers for background, external markers, are obtained by eroding the resulted image, as the complement of the image of internal markers.

\subsection{An application example: Microscopic bone marrow biopsies}

To evaluate the two marker algorithms that improve segmentation when using WT, we processed and analyzed microscopic images from bone marrow biopsies. Mainly these images are composed by three tissues: fat, hematopoietic tissue and bone trabecular tissue. Bone trabecular tissue is required to be segmented for diagnosis support. The complexity of these samples has motivated the development of this work.

The analyzed images were obtained through an optic microscope Medicux-12 with a CCD Hitachi KP-C550 camera. A dye composed of hematocylin and eosyn was applied with the objective to highlight the bone trabeculae. A set of 47 images taken from fourteen patients' biopsies were processed. The resolution of the images is $640 \times 480$ pixels and 256 gray levels.

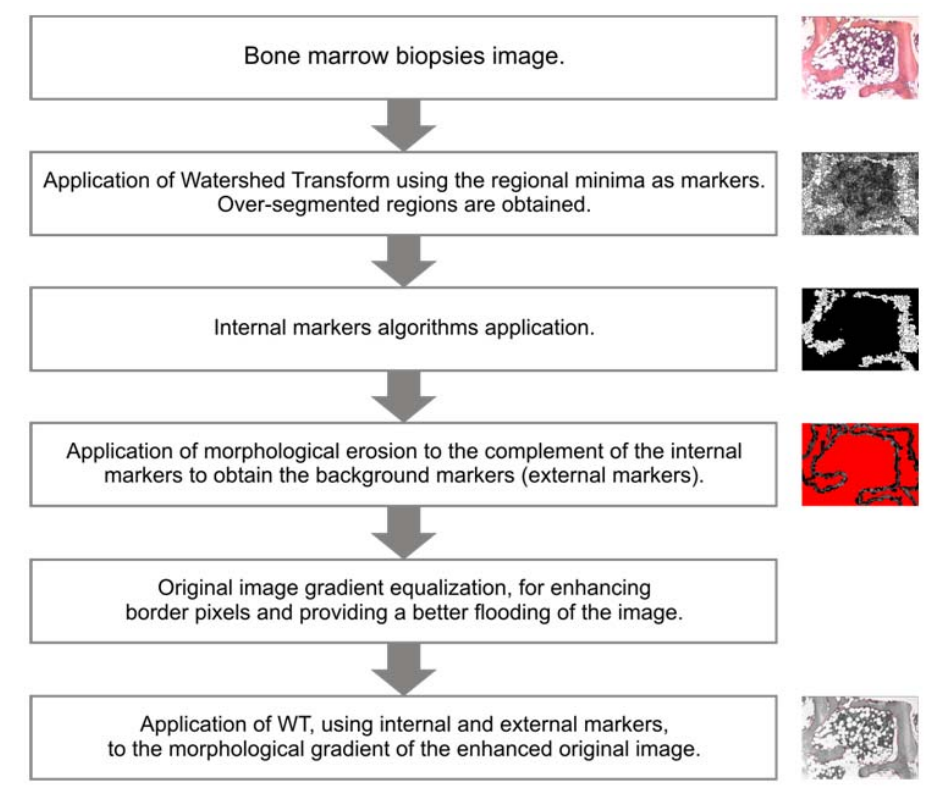

Figure 1. Steps of algorithms applied to bone marrow biopsies segmentation.

Images were acquired through a microscope where light, resolution and contrast were established by the lab technician to obtain the best visualization possible. Due to the great variability of these conditions it is impossible to define markers automatically with the methods developed so far.

Algorithms were implemented in Matlab ${ }^{\circledR}$ R14. We worked with the standard functions of this language and Mathematical Morphology and Fuzzy Logic Toolboxes.

Figure 1 shows the final algorithms steps applied to trabeculae images.

\section{Error analysis}

Pathologist determined the gold standard segmentation by marking manually the regions corresponding to bone trabecular tissue. We determined indices in order to estimate the segmentation error of each algorithm and its different parameters ${ }^{[19]}$. 
We compute mean classification error over the 47 test images with different pathologies and microscope images characteristics.

We varied threshold, membership functions, mean and standard deviation, and fuzzy operators for the fuzzy logic based algorithm. We varied median filter window size for clustering algorithm.

There are different methods for error analysis, in order to compare the performance of different algorithms. Next cases are likely to appear:

True positive (TP): a pixel that belongs to the expert segmented region and was detected as "object-of-interest” by the algorithm;

True negative (TN): a pixel that does not belong to the expert segmented region and was detected as "non-object-of-interest" by the algorithm;

False positive (FP): a pixel that does not belong to the expert segmented region and was detected as "object-of-interest" by the algorithm;

False negative (FN): a pixel that belongs to the expert segmented region and was detected as "non-object-of-interest” by the algorithm.

The numbers of false positives, false negatives, true positives and true negative were obtained.

From these four values, other indicators were calculated to analyze segmentation accuracy, its reliability and predictive power of distinguishing between positive and negative cases for analysis. False negative rate (FNR) is defined as:

$$
F N R=\frac{F P}{F P+T N}
$$

where the denominator indicates the total of negative cases.

False positive rate (FPR) is defined as:

$$
F P R=1-\frac{F N}{F N+T P}
$$

corresponding to the ratio between the number of positive cases that were not identified to the number of total positive cases.

Sensitivity (SE) measures the accuracy of the classifier to recognize positive cases and Specificity (SP) measures the accuracy of the classifier to recognize negative cases; they are defined as:

$$
S E=\frac{T P}{T P+F N} ; S P=\frac{T N}{T N+F P}
$$

We used ROC curves to determine an appropriate value for the algorithms' parameters. These graphs plot FP vs. TP. Area under the curve is a good indicator of algorithms performance for recognizing positive cases and negative cases simultaneously. We compare these curves and measure their area to assess algorithms' performances and to select the best algorithm parameters ${ }^{[19]}$. 


\section{Results}

The marker definition algorithm based in clustering requires a filtering process. WT over-segmented regions size is determined by the enhancement filter mask size. The mask size for clustering algorithmwas found heuristically by computation of segmentation error indicators. We found that minimal mean error is achieved using a median filter $3 \times 3$ pixel mask, as can be seen in Table 1.

Table 1. Clustering based algorithm errors using different filter mask sizes.

\begin{tabular}{llllllll}
\hline & \multicolumn{9}{c}{ Filter mask size } \\
\cline { 2 - 8 } & $\mathbf{1 \times 1}$ & $\mathbf{2 \times 2}$ & $\mathbf{3 \times 3}$ & $\mathbf{4 \times 4}$ & $\mathbf{5 \times 6}$ & $\mathbf{6 \times 6}$ & $\mathbf{7 \times 7}$ \\
\hline Clustering algorithm error & 0.2411 & 0.1603 & 0.1179 & 0.1832 & 0.2028 & 0.2143 & 0.2941 \\
FN & 0.1865 & 0.1035 & 0.0868 & 0.0914 & 0.0535 & 0.0276 & 0.1852 \\
FP & 0.0546 & 0.0567 & 0.0310 & 0.0918 & 0.1494 & 0.1867 & 0.1089 \\
TN & 0.3173 & 0.8342 & 0.7991 & 0.7272 & 0.3918 & 0.4456 & 0.2949 \\
TP & 0.4415 & 0.0054 & 0.0830 & 0.0897 & 0.4054 & 0.3401 & 0.4111 \\
Specificity & 0.8532 & 0.9363 & 0.9626 & 0.8879 & 0.7239 & 0.7047 & 0.7303 \\
Sensitivity & 0.2969 & 0.9499 & 0.5114 & 0.5049 & 0.1165 & 0.0750 & 0.3106 \\
\hline
\end{tabular}

For Fuzzy Logic based algorithm, we tested two different operators: product logic and compensatory fuzzy logic. We also tested different membership definitions and threshold values.

When analyzing fuzzy operators, the compensatory logic presented a lower error in all the 47 tested images. The mean value of the error was 0.102 and the standard deviation 0.07 . When using probabilistic operators, the mean value of the error was 0.112 and the standard deviation 0.059 . In both cases, the expert indicates the object of interest in order to determine membership functions. Binarization were done using OTSU algorithm.

The analysis of the errors, confirms the results obtained in previous works: the segmentation mean error increases when membership function parameters are fixed for all images. Consequently, the best strategy for this kind of images is: 1) selection of object of interest by the expert; 2) membership functions determination and 3) evaluation of the compensatory fuzzy logic operator. This membership functions, constructed using the mean value and standard deviation of only one region, presented very satisfactory results. Since histograms present a great variability, it is not possible to defining a unique membership function for all the images.

Table 2. Fuzzy Logic based algorithm errors. Column 1: Errors using a fixed degree-of-truth threshold for the predicate. Column 2: Using OTSU threshold.

\begin{tabular}{lll}
\hline & Fixed threshold & OTSU threshold \\
\hline Algorithm error & 0.1348 & 0.1025 \\
TP & 0.1302 & 0.0190 \\
TN & 0.7349 & 0.8654 \\
FP & 0.0675 & 0.0551 \\
FN & 0.0673 & 0.0473 \\
Specificity & 0.9219 & 0.9411 \\
Sensitivity & 0.3408 & 0.7132 \\
\hline
\end{tabular}

When considering "object" or "non-object", we tested different thresholds for the predicate degrees of truth. For these tests, first we fixed membership functions and logic operators as well. We sweep this threshold from zero to one, by step of 0.05 and took the value that gave the minimum error (Table 2, column 1). Even using the best threshold, errors were not 
satisfactory, confirming the results obtained in previous works, where lower errors were obtained using OTSU threshold. The Fuzzy-based algorithm presented lower error, higher specificity and sensitivity than clustering algorithm (Table 2, column 2). Another kind of image might require another error analysis.

Figure 2 shows ROC curves obtained by both algorithms. It can be observed, that fuzzy logic based algorithm shows a better performance than clustering algorithm when considering the area under the curve.
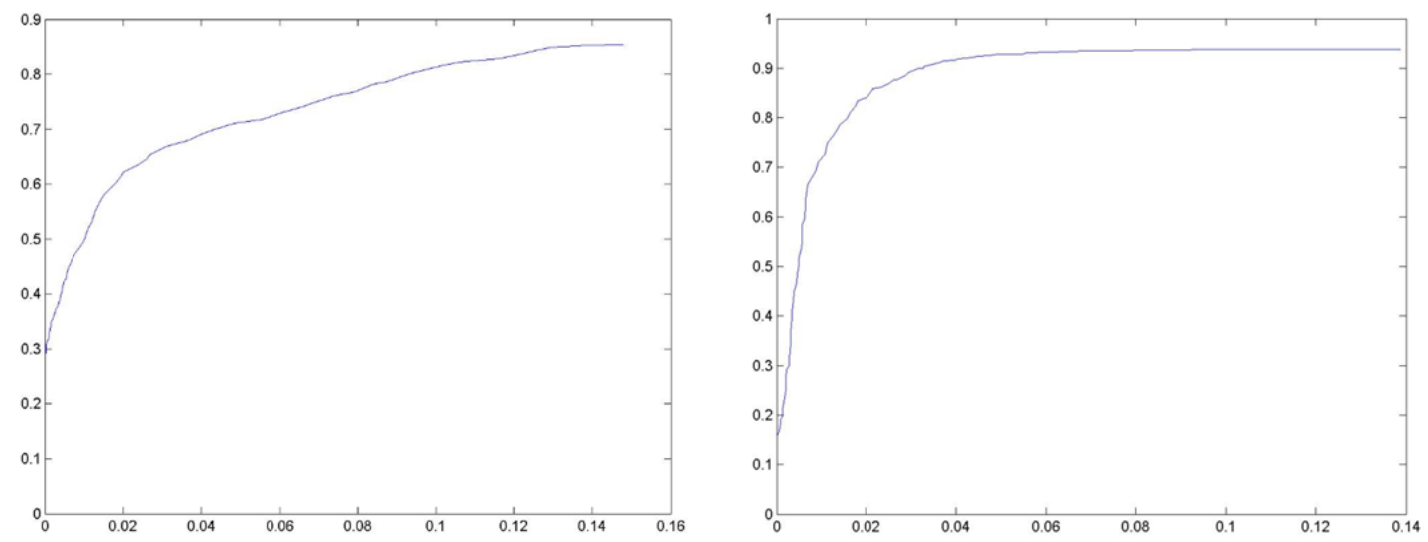

Figure 2. Average ROC curves for clustering algorithm (left) and fuzzy logic algorithm (right) for marker determination.

Figure 3 shows final segmentation results when the best marker definition algorithm was applied in typical bone marrow biopsies.
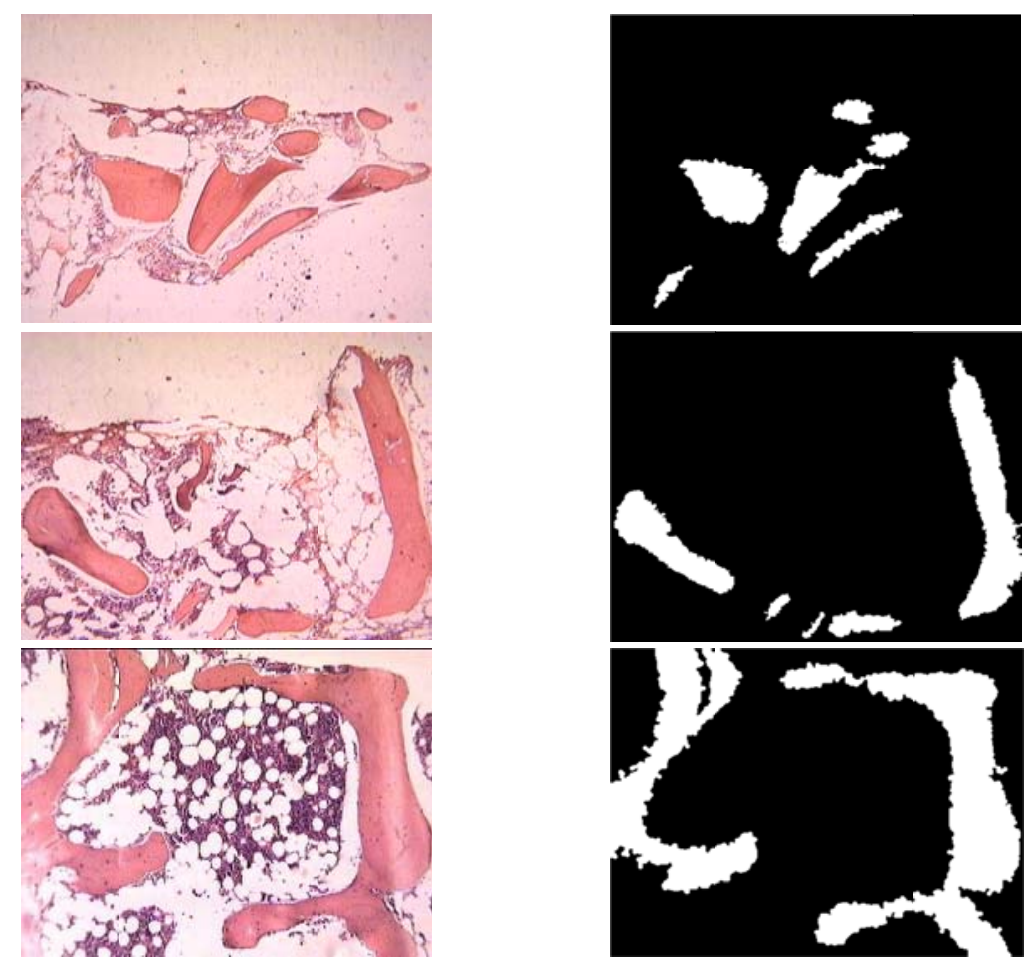

Figure 3. Bone marrow biopsies trabeculae segmentation. Left: original images. Right: trabeculae zones (white) and non-trabecuale zones (black). 


\section{Discussion}

We obtained different regions by applying the WT to the original image, obtaining an over-segmented image into regions. Gray level mean and standard deviation of each region characterize regions corresponding to different components in the images. The $k$-means algorithm and fuzzy logic based algorithms were applied to the features in those regions, determining effectively the internal markers of the objects. We applied morphological operators to the class that represents the objects to generate the internal and markers. Image gradient was enhanced for a new application of the WT.

The classification by using over-segmented regions has proved to be advantageous. Firstly, it reduces the noise present in the medical images, thus achieving a more precise and acute segmentation. Furthermore, it reduces the computational cost of the algorithm due to the low quantity of pixels to be analyzed. This characteristic is important due to high spatial resolution of medical images. Another advantaged of using the over segmentation regions is that is not need a window statistics analysis to determine membership function parameters, because each region mean value and standard deviation is enough to obtain gray level statistics.

Trabeculae regions from biopsies of different patients and pathologies could be successfully segmented by the application of CFL, improving the acquired segmentations with conventional fuzzy logic. The proposed algorithms are versatile and they could be applied to other type of images. These features made the algorithm adaptable to any particular image without modifying either the algorithm or its parameters.

The error against manually segmented images and ROC analysis allowed parameter definition and in consequence improved the accuracy and global segmentation performance analysis. Although the best performance algorithm, based in CFL logic, is not fully automatic, it presented a good adaptation to the great complexity and variability of bone marrow biopsies images, as an example of typical medical images.

Future works are oriented in testing a different set of images, particularly those which require new features from the over-segmentedregions.

\section{References}

[1] Gauch J M. Image segmentation and analysis via multiescale gradient watershed hierarchies, IEEE Trans. on Image Processing. 1999; 8-1.

[2] Li P and Xiao X. An unsupervised marker image generation method for watershed segmentation of multiespectral imagery, Geoscience Journal. 2004; 8(3): 325-331. http://dx.doi.org/10.1007/BF02910252

[3] Hernandez S E, Barner K E. Joint region merging criteria for Watershed-Based image segmentation. IEEE Trans on Image Processing. 2000. PMid:18255372

[4] Stanislav L S, Straber W. Extracting regions of interest applying a local watershed transformation. Proc. of IEEE Visualization. 2000.

[5] Zadeh L, Conjuntosdifusos. Información y Control. 1965; 8: 338-353. http://dx.doi.org/10.1016/S0019-9958(65)90241-X

[6] Zadeh L, A partir de cálculos con números a la computación con palabras de la manipulación de lasmediciones a la manipulación de laspercepciones, en RevistaInternacional de MatemáticaAplicada y Ciencias de la Computación. 2002; 12(3): 307-324.

[7] Gonzalez M A, Cuadrado T R, Ballarin V L. Comparing marker definition algorithms for Watershed segmentation in microscopy images, Journal of Computer Science and Technology. 2008; 8(3): 151-157.

[8] Gonzalez M A, Ballarin V L, Segmentación de imágenesutilizando la Transformada Watershed: Obtención de marcadoresmedianteLógicaDifusa, IEEE America Latina. 2008; 6(2): 223-228.

[9] Gonzalez M A, Ballarin V L, Automatic marker determination for watershed segmentation using clustering Latin American Applied Research. 2009; 39(3): 225-229.

[10] Vincent L and Soille P, Watersheds in digital spaces: An efficient algorithm based on immersion simulations, IEEE trans. Pattern Anal, Machine Intell. 1991; 13(6): 583-598. http://dx.doi.org/10.1109/34.87344

[11] Beucher F, Meyer F, The morphological approach to segmentation: The Watershed Transformation, Mathematical Morphology and its application to image processing, E. R. Dougherty, Ed. New York: Marcel Decker. 1993; 12: 433-481. 
[12] Espin Andrade R A, Suarez A R and Gomez J M A general approach for knowledge discovery based on compensatory fuzzy logic and metaheuristic search, Universidad de la Havana, Cuba, 2000.

[13] Wang D A, Multiscale gradient algorithm for Image Segmentation using Watersheds, Pattern Recognition. 1997; 30: $2043-2052$. http://dx.doi.org/10.1016/S0031-3203(97)00015-0

[14] Cheng H D,Chen Y H, Sun Y, A novel fuzzy entropy approach to image enhancement and thresholding. Signal Processing. 1999; 75: 277-301. http://dx.doi.org/10.1016/S0165-1684(98)00239-4

[15] Ceccarelli M, Musacchiar S, Petrosino A, A fuzzy scale-space approach to feature-based image representation and retrieval, Lecture Notes in Computer Science. 2004; 3704: 377-385. http://dx.doi.org/10.1007/11565123_36

[16] Ceccarelli M, Petrosino A. A parallel fuzzy scale-space approach to the unsupervised texture separation. Pattern Recognition Letters. 2002; 23: 557-567. http://dx.doi.org/10.1016/S0167-8655(01)00151-9

[17] Mc Queen J B, Some Methods for Classification and Analysis of Multivariate Observation. Proceedings of the 5th Berkeley Symposium on Mathematical Statistics and Probability, Berkeley University of California Press. 1967; 281-297.

[18] Otsu Nobuyuki. A threshold selection method from grey level histograms. In: IEEE Transactions on Systems, Man, and Cybernetics. New York.1979; 62-66.

[19] Fawcett T, ROC Graphs: Notes and Practical Considerations for Researchers. Technical report. Palo Alto, USA: HP Laboratories 2004. 